\title{
High-order time expansion path integral ground state
}

\author{
R. Rota, J. Casulleras, F. Mazzanti, and J. Boronat \\ Departament de Física i Enginyeria Nuclear, Campus Nord B4-B5, Universitat Politècnica de Catalunya, E-08034 Barcelona, Spain
}

(Received 23 July 2009; published 22 January 2010)

\begin{abstract}
The feasibility of path integral Monte Carlo ground state calculations with very few beads using a high-order short-time Green's function expansion is discussed. An explicit expression of the evolution operator which provides dramatic enhancements in the quality of ground-state wave functions is examined. The efficiency of the method makes possible to remove the trial wave function and thus obtain completely model-independent results still with a very small number of beads. If a single iteration of the method is used to improve a given model wave function, the result is invariably a shadow-type wave function, whose precise content is provided by the high-order algorithm employed.
\end{abstract}

DOI: 10.1103/PhysRevE.81.016707

PACS number(s): 02.70.Ss, 31.15.xk

Quantum Monte Carlo path integral calculations provide a powerful approach to many-body physics both at zero [1-3] and finite temperatures [4]. They rely on using the classical action of the system in imaginary time to define a path distribution probability following Feynman's approach. For systems with Bose statistics, the probability is positive and the only approximation involved in the calculations comes from the discretization of time, which prevents an exact evaluation of the action. In path integral calculations, chains with large numbers of time slices or beads are just a realization of these ideas [5]. If an accurate evaluation of the action for paths with large time steps were possible, the complexity of path integral calculations and, in particular, ergodicity issues, would be greatly reduced.

For ground-state calculations, the path integral groundstate (PIGS) approach [1-3] provides a method to systematically improve a trial wave function, by repeated application of the evolution operator in imaginary time, which eventually drives the system into the ground state [6]. Here again, if an accurate implementation of the evolution operator for large time steps were available, it could be used to build variational wave functions of very high quality.

In this paper, we focus on high-order short-time expansions of the Green's function, which have been the subject of a series of works in recent years [7-9]. This progress has led to a deep understanding of their properties and provided various extremely accurate decompositions of the evolution operator $\hat{U}=\exp (\varepsilon \hat{H})$, with $\hat{H}=\hat{T}+\hat{V}$, expressed as products of unitary operators of the basic components $\hat{U}=\exp (\varepsilon \hat{T})$ and $\hat{U}=\exp (\varepsilon \hat{V})$. An important result is that the use of a finite time step $\varepsilon$ can be renormalized in the potential. This means that for a range of time steps $\varepsilon$, one is in practice virtually free from finite time step errors. As a consequence, one can successfully use very few instances of the resulting imaginary time propagator to wipe out any excited state component from an initial variational wave function. This allows us to obtain an accurate description of the exact ground state wave function with little numerical effort. In this work we show that this goal can be easily achieved even when the starting variational wave functions contains no more additional information than the bosonic statistics, that is, when one starts from $\Psi_{m}=1$. This is a relevant feature of the method as it allows us to get accurate information about a physical system when no a priori knowledge about the ground state wave function is available.

We make use of the decomposition schemes proposed by Chin $[9,10]$ in order to evaluate the feasibility of PIGS calculations with high-order propagators using very few beads $\left(N_{b}\right)$. To this end, we revisit the ground state of bulk superfluid ${ }^{4} \mathrm{He}$. We also explore the possibility of performing ground-state calculations of boson systems without any model wave function, i.e., to take as a starting point $\Psi_{\mathrm{m}}=1$ and rely in the propagator's quality to build the actual wave function exclusively from the Hamiltonian. It is also interesting to note that if one does make use of a trial wave function, the application of Chin's evolution operator $[9,10]$ produces a much enhanced model that actually incarnates a shadow wave function [6]. In this way, Chin's analysis provides a deep understanding of the success of shadowlike wave functions $[11,12]$ and sheds light onto the actual mechanisms leading to this kind of wave functions.

Decompositions of the evolution operator preserving unitarity in the form,

$$
\exp [\varepsilon(\hat{T}+\hat{V})]=\prod_{i=1}^{N} \exp \left(t_{i} \varepsilon \hat{T}\right) \exp \left(v_{i} \varepsilon \hat{V}\right)+\mathcal{O}\left(\varepsilon^{n+1}\right),
$$

are the starting point which have culminated in the algorithms due to Chin that we test here. By means of a proper selection of the factorization coefficients $\left\{t_{i}\right\}$ and $\left\{v_{i}\right\}$, any given order can be achieved [the resulting expression is $n$th order since the effective Hamiltonian is then $\left.\hat{T}+\hat{V}+\mathcal{O}\left(\varepsilon^{n}\right)\right]$. However, if some of the $t_{i}$ factorization coefficients are negative, involving diffusion processes backward in time, the algorithm cannot be used in the context of quantum Monte Carlo calculations. Fourth-order algorithms, for which Chin has worked out a complete characterization [9], happen to be somehow unique, as for only forward decompositions, the highest order that can be achieved is four. These developments result from a careful use of the expansion

$$
\begin{aligned}
& \prod_{i=1}^{N} \exp \left(t_{i} \varepsilon \hat{T}\right) \exp \left(v_{i} \varepsilon \hat{V}\right) \\
& =\exp \left\{\varepsilon e_{T} \hat{T}+\varepsilon e_{V} \hat{V}+\varepsilon^{2} e_{T V}[\hat{T}, \hat{V}]+\varepsilon^{3} e_{T T V}[\hat{T},[\hat{T}, \hat{V}]]\right. \\
& \left.\quad+\varepsilon^{3} e_{V T V}[\hat{V},[\hat{T}, \hat{V}]]\right\}+\cdots,
\end{aligned}
$$


which allows us to keep only the simplest term $[\hat{V},[\hat{T}, \hat{V}]]$, which produces a sort of renormalized potential,

$$
[\hat{V},[\hat{T}, \hat{V}]]=\frac{\hbar^{2}}{m} \sum_{i=1}^{N}\left|\boldsymbol{F}_{i}\right|^{2}
$$

with $\boldsymbol{F}_{i}=\sum_{j \neq i}^{N} \nabla_{i} V\left(r_{i j}\right)$. A remarkable characteristic of the resulting expansions is that they lead to a continuous family of fourth-order algorithms characterized by one free parameter. It is interesting to note that with a proper choice of that parameter $\left[t_{1}\right.$ in Eq. (4) $]$ one is able to fine tune the leading error term of the propagator to any desired value, including changing sign in a controlled way. Thus, one is enabled to minimize the value of the fourth-order error coefficient, and in doing so, even try to cancel, to the largest possible extent, the contributions of the next orders. In practice, this means that a particular value of the free parameter entering in the algorithm produces exceedingly accurate and stable results. A different strategy to improve the order of the propagator is the use of a multiproduct expansion with a controlled violation of positivity [13].

Among the various decompositions proposed in Ref. [9], the particular scheme chosen in this work is

$$
e^{-\tau \hat{H}}=e^{-v_{0} \tau \hat{V}} e^{-t_{1} \tau \hat{T}} e^{-v_{1} \tau \hat{W}} e^{-t_{2} \tau \hat{T}} e^{-v_{1} \tau \hat{W}} e^{-t_{1} \tau \hat{T}} e^{-v_{0} \tau \hat{V}},
$$

with $\hat{W}=\hat{V}+\left(u_{0} / v_{1}\right) \tau^{2}[\hat{V},[\hat{T}, \hat{V}]]$.

The various factorization coefficients are all dependent on the single free parameter $t_{1}$ [9]. The range of possible values for $t_{1}$ is $0 \leq t_{1} \leq 0.5$ and experience shows that the optimal value is nearly independent of $\tau=\varepsilon / N_{b}$. A similar decomposition has been recently used in path integral Monte Carlo (PIMC) simulations at finite temperature showing high accuracy [14].

The operator splitting (4) allows for an estimation of the Green's function $G(R, S, \tau)=\left\langle R\left|e^{-\tau H}\right| S\right\rangle$, which in turn provides the mechanism for building accurate wave functions starting from a trial wave function, $\Psi(R)$ $=\int G(R, S, \tau) \Psi_{\mathrm{m}}(S) d S$. More explicitly,

$$
\begin{aligned}
\Psi(R)= & \int e^{-v_{0} \tau V(R)} e^{-\left(R-S_{1}\right)^{2} / 4 D t_{1} \tau} e^{-v_{1} \tau W\left(S_{1}\right)} \\
& \times e^{-\left(S_{1}-S_{2}\right)^{2} / 4 D t_{2} \tau} e^{-v_{1} \tau W\left(S_{2}\right)} e^{-\left(S_{2}-S\right)^{2} / 4 D t_{1} \tau} \\
& \times e^{-v_{0} \tau V(S)} \Psi_{\mathrm{m}}(S) d S_{1} d S_{2} d S .
\end{aligned}
$$

Actually, Eq. (5) sets the grounds for building wave functions consistent with the decomposition of the propagator (4). It has an intuitive content too: it states that the actual value of $\Psi(R)$ should be taken as a weighted average of neighbor shadow values $\Psi(S)$, with a weight given by a precise combination of exponentials of the (finite time step renormalized) potential. Moreover, it is also very suggesting considering the double possibility that Eq. (5) offers, either as a powerful enhancement of an a priori model wave function $\Psi_{\mathrm{m}}(R)$ or directly as a tool to generate the wave function directly from the Hamiltonian.

Equation (5) can also be written in terms of relative rather than absolute auxiliary coordinates,

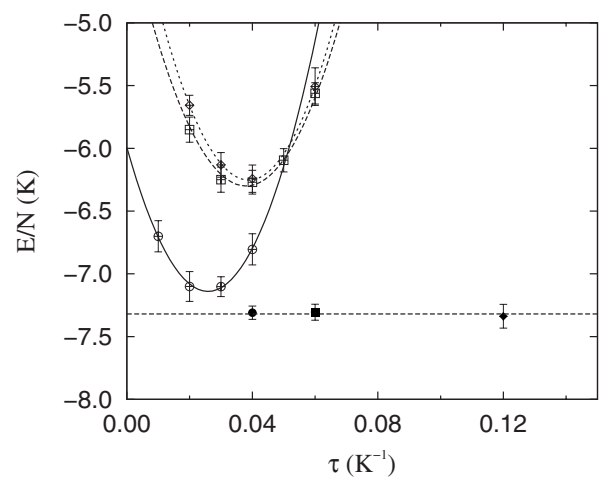

FIG. 1. Variational energies obtained with a single time step $\tau$ and the proposed wave function. Open circles, squares, and diamonds correspond to $\Psi_{\mathrm{m}}(R)$ of McMillan type, semiclassical model, and 1, respectively. The corresponding filled symbols stand for the asymptotic values using the three models and more than one time step.

$$
\begin{aligned}
\Psi(R)= & \int e^{-v_{0} \tau V(R)} e^{-\left(S_{1}^{2} / 4 D t_{1} \tau\right)} e^{-v_{1} \tau W\left(R+S_{1}\right)} e^{-\left(S_{2}^{2} / 4 D t_{2} \tau\right)} \\
& \times e^{-v_{1} \tau W\left(R+S_{1}+S_{2}\right)} e^{-\left(S^{2} / 4 D t_{1} \tau\right)} e^{-v_{0} \tau V\left(R+S_{1}+S_{2}+S\right)} \\
& \times \Psi_{\mathrm{m}}\left(R+S_{1}+S_{2}+S\right) d S_{1} d S_{2} d S
\end{aligned}
$$

While Eqs. (5) and (6) are equivalent, they lead to different estimators for the kinetic energy. The action of the kinetic operator on $\Psi(R)$ in Eq. (6) involves derivatives of $\Psi_{\mathrm{m}}$, in contrast to what happens when the prescription given in Eq. (5) is used.

It is possible to use the propagator $G(R, S, \tau)$ in order to improve the quality of any given trial wave function, with reduced variance in direct proportion to its quality. In fact, Eq. (6) satisfies the principle of zero variance: as the trial wave function $\Psi_{\mathrm{m}}$ approaches the exact ground state wave function, the propagation time $\tau$ can be continuously tuned to smaller values, with the $S$ distribution approaching a Dirac delta and $\Psi(R)$ and its derivatives approaching the exact ones.

In order to test the accuracy of methods (5) and (6), we have applied it to liquid ${ }^{4} \mathrm{He}$, a classical benchmark in quantum many-body physics. The calculations have been carried out at the experimental equilibrium density $\rho=0.365 \sigma^{-3}$ ( $\sigma$ $=2.556 \AA$ ) with $N=64$ atoms and using the HFD-B(HE) Aziz potential [15], which has proven to be highly accurate in the description of the experimental equation of state [16]. As a trial wave function, we use a simple Jastrow form based on the McMillan model, $\Psi_{\mathrm{m}}(R)=\prod_{i j} \exp \left[-0.5\left(b / r_{i j}\right)^{5}\right]$ with $b=1.20 \sigma$, and two additional variational parameters, $t_{1}$ and $\tau$ (5). After a quick search we found the optimal value $t_{1}$ $=0.35$ which was kept fixed for the rest of the calculations. The dependence of the variational energy on the remaining variational parameter $\tau$ is shown in Fig. 1, where the variational character of the calculation is clear. The horizontal axis corresponds to the $\tau$ parameter in $\mathrm{K}^{-1}$ units and the vertical axis represents the total energy per particle in $\mathrm{K}$. The data displayed as empty circles is the variational energy for the wave function based on the high-order action (5). The 
TABLE I. Total and potential energies per particle as a function of the imaginary time $\varepsilon$ and number of beads $N_{b}$ using different models for the initial wave function $\Psi_{\mathrm{m}}(R)$. Figures in parentheses are the statistical errors.

\begin{tabular}{|c|c|c|c|c|c|c|c|}
\hline \multirow[b]{2}{*}{$\begin{array}{c}\varepsilon \\
\left(\mathrm{K}^{-1}\right)\end{array}$} & \multirow[b]{2}{*}{$N_{b}$} & \multicolumn{2}{|c|}{ McMillan } & \multicolumn{2}{|c|}{ Semiclassical } & \multicolumn{2}{|c|}{1} \\
\hline & & $\begin{array}{c}E / N \\
(\mathrm{~K})\end{array}$ & $\begin{array}{c}V / N \\
(\mathrm{~K})\end{array}$ & $\begin{array}{c}E / N \\
(\mathrm{~K})\end{array}$ & $\begin{array}{c}V / N \\
(\mathrm{~K})\end{array}$ & $\begin{array}{c}E / N \\
(\mathrm{~K})\end{array}$ & $\begin{array}{c}V / N \\
(\mathrm{~K})\end{array}$ \\
\hline 0.01 & 1 & $-6.860(58)$ & $-20.924(30)$ & $-3.878(76)$ & $-17.246(44)$ & $-2.81(9)$ & $-17.009(52)$ \\
\hline 0.02 & 2 & $-7.175(51)$ & $-21.106(37)$ & $-6.234(76)$ & $-20.285(44)$ & $-5.73(12)$ & $-20.148(42)$ \\
\hline 0.04 & 4 & $-7.268(44)$ & $-21.413(35)$ & $-7.121(67)$ & $-21.284(43)$ & $-6.93(11)$ & $-21.186(39)$ \\
\hline 0.06 & 4 & $-7.303(35)$ & $-21.538(44)$ & $-7.306(64)$ & $-21.547(40)$ & $-7.15(11)$ & $-21.570(31)$ \\
\hline 0.08 & 6 & $-7.299(41)$ & $-21.534(38)$ & $-7.290(55)$ & $-21.583(39)$ & & \\
\hline 0.08 & 8 & & & & & $-7.25(8)$ & $-21.381(20)$ \\
\hline 0.10 & 8 & & & & & $-7.33(8)$ & $-21.498(12)$ \\
\hline 0.12 & 10 & & & & & $-7.34(9)$ & $-21.524(10)$ \\
\hline
\end{tabular}

position of the minimum is a compromise between a large $\tau$ value suitable for a large suppression of the excited components present in $\Psi_{\mathrm{m}}(R)$, and a small one suitable for a proper behavior of the variational wave function (5), which relies itself on a short-time expansion. The minimum is located at $\tau=0.025$ and the variational energy obtained is $E=-7.10 \mathrm{~K}$, only $\sim 0.2 \mathrm{~K}$ higher than the exact value.

Equally impressive is the data displayed as empty diamonds, which is a variational calculation using the same propagator (5) acting on $\Psi_{\mathrm{m}}(R)=1$, i.e., it is a variational calculation in which only the Hamiltonian and the statistics are used. We see in this case that the compromise between a large suppression of the nonground state components and a small $\tau$ parameter has been shifted toward a higher value $\tau$ $=0.04$, as could be expected. A third model for $\Psi_{\mathrm{m}}(R)$ consists in a semiclassical approximation, $\Psi_{\mathrm{m} \text {-sc }}(R)=\Pi_{i<j} \exp$ $\left[-\varepsilon V_{\mathrm{sr}}\left(r_{i j}\right)\right]$, with $V_{\mathrm{sr}}(r)=1 / r^{12}$ corresponding to the $r$ dependence of the Lennard-Jones potential around the core. This third model does not contain any free variational parameter since $\varepsilon$ is the total imaginary time of the propagator. The results obtained with $\Psi_{\mathrm{m} \text {-sc }}(r)$ (open squares) only improve slightly the variational energy obtained with $\Psi_{\mathrm{m}}(R)=1$.

It is also possible to apply several times the propagator to $\Psi_{\mathrm{m}}(R)$ in order to obtain better variational results and finally projecting out any excitation present in the model wave functions. This is shown in Fig. 1 with a filled circle point, which corresponds to the propagator applied $N_{b}=4$ times onto the Jastrow-McMillan wave function $\Psi_{\mathrm{m}}(R)$ for a total time propagation $\varepsilon=0.04 \mathrm{~K}^{-1}$. The same figure shows with a filled diamond the result of acting with $N_{b}=10$ propagators on $\Psi_{\mathrm{m}}(R)=1$ for a total time propagation $\varepsilon=0.12 \mathrm{~K}^{-1}$. Between these two points, the semiclassical model achieves convergence very fast with $N_{b}=4$ and time $\varepsilon=0.06 \mathrm{~K}^{-1}$.

The high-order Green's function can also be used to obtain the total energy following the standard procedure of evaluating the local energy of the trial wave function $\Psi_{\mathrm{m}}(R)$ at one end of the chain, and its high accuracy translates again in the need for a very small number of beads until convergence is achieved. The results obtained for the total and potential energies per particle are reported in Table I as a function of the total imaginary time $\varepsilon$. The potential energies are calculated in the center of the chain to remove any possible bias coming from $\Psi_{\mathrm{m}}(R)$; the total energies are estimated in the extreme, except for the case $\Psi_{\mathrm{m}}(R)=1$ where they are sampled in the center. The number of beads for a given time $\varepsilon$ is determined simply by the requirement that doubling its number (and simultaneously halving the propagation time per bead $\tau$ ) does not have any effect, which turns to be equivalent to keep the propagation time per bead below $\tau$ $=0.15 \mathrm{~K}^{-1}$. One would expect that this regime corresponds to keeping the finite time step error just below the detectable level. This is in accordance with Fig. 1, where we see that a propagation time per bead near $0.15 \mathrm{~K}^{-1}$ is close to the maximum value before the time step error starts to become apparent by bending upwards the variational energy curve. When both requirements: $\tau=\varepsilon / N_{b}$ to be small enough ( $\tau \leq 0.15 \mathrm{~K}^{-1}$ in our case) and $N_{b}$ large enough are met, the energy becomes independent of both $\varepsilon$ and $N_{b}$ and a good estimation of the ground state energy is achieved.

The results of Table I show that the convergence is quickly achieved with only a few number of beads: $N_{b}=4$ for the McMillan and semiclassical Jastrow factors, and $N_{b}=8$ for $\Psi_{\mathrm{m}}(R)=1$. Concerning the convergence for the potential energy, one can see in Table I that the exact (asymptotic in $\varepsilon$ ) value is independent of the trial wave function and its value is reached with only $N_{b}=4$. It should be taken into account however that, as in any PIGS method, the total length of the chain corresponding to $\left|\Psi_{\mathrm{m}}(R)\right|^{2}$ is twice that of $\Psi_{\mathrm{m}}(R)$, and that one $\tau$ propagator (5) involves three internal shadows.

Unbiased (pure) estimations of operators $\hat{O}$ other than the Hamiltonian can only be calculated in the center of the chain, $\langle\hat{O}\rangle=\mathcal{N}^{-1}\left\langle\Psi_{\mathrm{m}}|G(\varepsilon / 2) \hat{O} G(\varepsilon / 2)| \Psi_{\mathrm{m}}\right\rangle$. This holds, for instance, for the potential energy reported in Table I and the two-body radial distribution function $g(r)$ shown in Fig. 2. The present PIGS results for $g(r)$ show an excellent agreement with experimental data [17] for the trial wave functions used in this work. Even when $\Psi_{\mathrm{m}}(R)=1$ the result is the same, the calculation requiring only a few more beads.

Another relevant function that can be computed in an unbiased way is the one-body density matrix $\rho(r)$, whose asymptotic limit is the condensate fraction. The calculation of $\rho(r)$ has been carried out by incorporating worm move- 


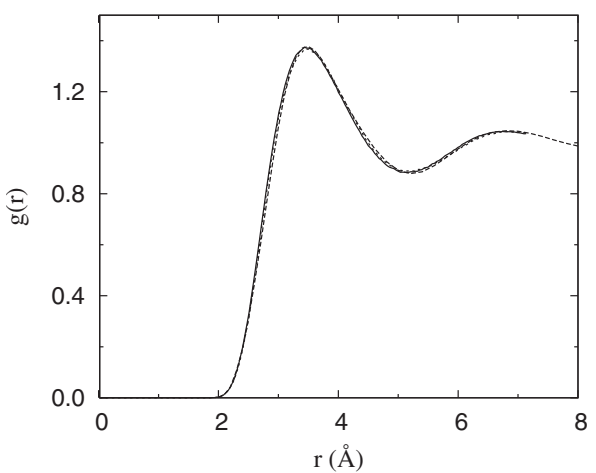

FIG. 2. Two-body radial distribution function $g(r)$. The solid and dotted lines correspond to present results using for $\Psi_{\mathrm{m}}(R)$ a Jastrow-McMillan factor or $\Psi_{\mathrm{m}}(R)=1$, respectively. The dashed line is the experimental data from Ref. [17].

ments in the sampling [18], a technique that has been developed for path integral Monte Carlo (finite temperature) and that we have extended to PIGS. One of the main advantages of this method is that $\rho(r)$ comes properly normalized, and thus eliminates any uncertainty introduced by the a posteriori normalization factor. In Fig. 3, results for $\rho(r)$ obtained using different trial wave functions $\Psi_{\mathrm{m}}$ are shown. As one can see, the results are statistically indistinguishable and predict a condensate fraction $n_{0}=0.080(2)$, in nice agreement with a recent PIMC estimation at $T=1 \mathrm{~K}$ $\left[n_{0}=0.081(2)\right][18]$.

Summarizing, the high-order short-time expansion of the Green's function presented in this work allows performing high quality variational calculations on systems for which no model of ground state wave function is known. Although variational in nature, the accuracy of the used propagator is so high that marginally small deviations from the exact ground state can be achieved with very few time steps. This

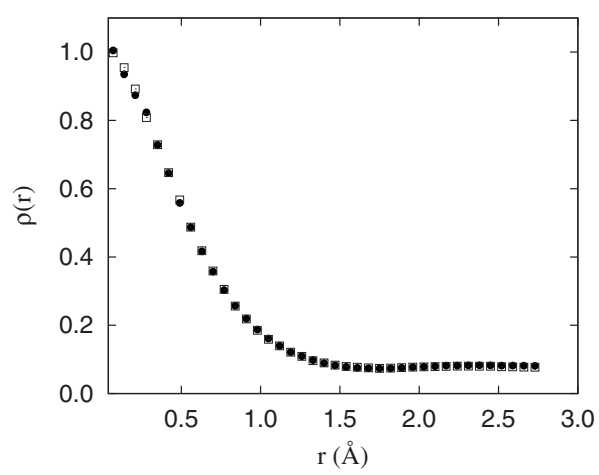

FIG. 3. One-body density matrix $\rho(r)$. Filled circles and open squares stand for PIGS results using for $\Psi_{\mathrm{m}}(R)$ a Jastrow-McMillan factor $\left(N_{b}=5\right)$ or $\Psi_{\mathrm{m}}(R)=1\left(N_{b}=10\right)$, respectively. The error bars are smaller than the size of the symbols.

is illustrated in the case of liquid ${ }^{4} \mathrm{He}$ at equilibrium density, where the propagator provides for $N_{b}=1$ and $\Psi_{\mathrm{m}}(R)=1$ a variational energy $E / N=-6.20 \mathrm{~K}$, and converges to the exact value already with $N_{b}=8$. When used to improve a Jastrow-McMillan wave function, it produces a shadow-like variational wave function for $N_{b}=1$ with a variational ground-state energy $E / N=-7.10 \mathrm{~K}$, only $\sim 0.2 \mathrm{~K}$ higher than the exact value. Repeated application of the propagator leads to variational results which are asymptotically exact for values as low as $N_{b}=4$. The prospects for future work are promising, since this opens the road to being able to obtain results for systems whose ground-state wave function is poorly known or even unknown.

We wish to thank stimulating discussions with Siu Chin. Partial financial support from DGI (Spain) Grant No. FIS2008-04403 and Generalitat de Catalunya Grant No. 2005SGR-00779 is also acknowledged.
[1] A. Sarsa, K. E. Schmidt, and W. R. Magro, J. Chem. Phys. 113, 1366 (2000).

[2] D. E. Galli and L. Reatto, Mol. Phys. 101, 1697 (2003).

[3] J. E. Cuervo, P.-N. Roy, and M. Boninsegni,J. Chem. Phys. 122, 114504 (2005).

[4] D. M. Ceperley, Rev. Mod. Phys. 67, 279 (1995).

[5] D. Chandler and P. G. Wolynes, J. Chem. Phys. 74, 4078 (1981).

[6] O. Ciftja and S. A. Chin, Phys. Rev. B 68, 134510 (2003).

[7] M. Takahashi and M. Imada, J. Phys. Soc. Jpn. 53, 3765 (1984).

[8] M. Suzuki, Phys. Lett. A 201, 425 (1995).

[9] S. A. Chin and C. R. Chen, J. Chem. Phys. 117, 1409 (2002).

[10] S. A. Chin, Phys. Rev. E 69, 046118 (2004)
[11] S. Vitiello, K. Runge, and M. H. Kalos, Phys. Rev. Lett. 60 , 1970 (1988).

[12] L. Reatto and G. L. Masserini, Phys. Rev. B 38, 4516 (1988).

[13] R. E. Zillich, J. Mayrhofer, and S. Chin, e-print arXiv:0907.3495.

[14] K. Sakkos, J. Casulleras, and J. Boronat, J. Chem. Phys. 130, 204109 (2009).

[15] R. A. Aziz, F. R. W. McCourt, and C. C. K. Wong,Mol. Phys. 61, 1487 (1987).

[16] J. Boronat and J. Casulleras, Phys. Rev. B 49, 8920 (1994).

[17] E. C. Svensson, V. F. Sears, A. D. B. Woods, and P. Martel, Phys. Rev. B 21, 3638 (1980).

[18] M. Boninsegni, N. V. Prokofev, and B. V. Svistunov, Phys. Rev. E 74, 036701 (2006). 